\title{
THE INFLUENCE OF SWIRL BRAKES ON THE ROTORDYNAMIC FORCES GENERATED BY DISCHARGE-TO-SUCTION LEAKAGE FLOWS IN CENTRIFUGAL PUMPS
}

\author{
Joseph M. Sivo, Allan J. Acosta, \\ Christopher E. Brennen, and Thomas K. Caughey \\ Department of Mechanical Engineering \\ California Institute of Technology \\ Pasadena, California
}

\section{ABSTRACT}

Increasing interest has been given to swirl brakes as a means of reducing destabilizing rotordynamic forces due to leakage flows in new high speed rocket turbopurnps. Although swirl brakes have been used successfully in practice (such as with the Space Shuttle HPOTP), no experimental tests until now have been performed to demonstrate their beneficial effect over a range of leakage flow rates. The present study investigates the effect of swirl brakes on rotordynamic forces generated by discharge-to-suction leakage flows in the annulus of shrouded centrifugal pumps over a range of subsynchronous whirl ratios and various leakage flow rates. In addition, the effectiveness of swirl brakes in the presence of leakage inlet (pump discharge) swirl is also demonstrated. The experimental data demonstrates that with the addition of swirl brakes a significant reduction in the destabilizing tangential force for lower flow rates is achieved. At higher flow rates, the brakes are detrimental. In the presence of leakage inlet swirl, brakes were effective over all leakage flow rates tested in reducing the range of whirl frequency ratio for which the tangential force is destabilizing.

\section{NOMENCLATURE}

\section{$\left[A^{*}\right] \quad$ Rotordynamic force matrix}

B Depth of logarithmic spiral channel on swirl vane

c Cross-coupled damping coefficient, normalized by $\rho \pi \omega^{2} R_{2}{ }^{2} L \varepsilon$

C Direct damping coefficient, normalized by $\rho \pi \omega^{2} R_{2}{ }^{2} L \varepsilon$

$F^{*}{ }_{x}(t) \quad$ Lateral horizontal force in the laboratory frame

$F^{*}{ }_{y}(t) \quad$ Lateral vertical force in the laboratory frame

$F^{*}$ or Steady horizontal force

$F^{*}$ oy Steady vertical force

$F^{*}{ }_{n} \quad$ Force normal to whirl orbit

$F_{n} \quad$ Force normal to whirl orbit normalized by $\rho \pi \omega^{2} R_{2}^{2} L \varepsilon$

$F^{*}$. Force tangent to whirl orbit

$F_{t} \quad$ Force tangent to whirl orbit normalized by $\rho \pi \omega^{2} R_{2}{ }^{2} L \varepsilon$

$F_{1}, F_{2} \quad$ Lateral forces in rotating frame

H Clearance between impeller shroud and housing

k Cross-coupled stiffness coefficient normalized by $\rho \pi \omega^{2} R_{2}^{2} L \varepsilon$

$\mathrm{K} \quad$ Direct stiffness coefficient normalized by $\rho \pi \omega^{2} R_{2}{ }^{2} L \varepsilon$

L Axial length of impeller

M Direct added mass coefficient normalized by $\rho \pi \omega^{2} R_{2}^{2} L \varepsilon$

Q Volumetric leakage flow rate

$R_{2} \quad$ Radius of impeller at leakage inlet

$u_{s} \quad$ Mean leakage inlet path velocity of fluid

$u_{\theta} \quad$ Mean leakage inlet swirl velocity of fluid 
$x^{*}(t) \quad$ Horizontal displacement of impeller on its orbit

$y^{*}(t) \quad$ Vertical displacement of impeller on its orbit

$\alpha \quad$ Turning angle of logarithmic spiral channel on swirl vane

$\Gamma \quad$ Leakage inlet swirl ratio, $u_{\theta} / \omega R_{2}$

$\varepsilon \quad$ Eccentricity of impeller's circular whirl orbit

$\rho \quad$ Density of leakage fluid

$\phi \quad$ Leakage flow coefficient, $u_{s} / \omega R_{2}$

$\omega \quad$ Main shaft radian frequency

$\Omega$

Whirl radian frequency

\section{INTRODUCTION}

Previous experimental and analytical results have shown that discharge-to-suction leakage flows in the annulus of a shrouded centrifugal pump contribute substantially to the fluid induced rotordynamic forces (Adkins, 1988). Experiments conducted in the Rotor Force Test Facility (RFTF) at Caltech on an impeller undergoing a prescribed whirl have indicated that the leakage flow contribution to the normal and tangential forces can be as much as $70 \%$ and $30 \%$ of the total, respectively (Jery, 1986). Recent experiments at Caltech have examined the rotordynamic consequences of leakage flows and have shown that the rotordynamic forces are functions not only of the whirl ratio but also of the leakage flow rate and the impeller shroud to pump housing clearance. The forces were found to be inversely proportional to the clearance and a region of forward subsynchronous whirl was found for which the average tangential force was destabilizing. This region decreased with flow coefficient (Guinzburg, 1992a).

The motivation for the present research is that the previous experiments have shown that leakage inlet (pump discharge) swirl can increase the cross-coupled stiffness coefficient (in some tests by over $100 \%$ ) and hence increase the range of positive whirl for which the tangential force is destabilizing. One might therefore surmise that if the swirl velocity within the leakage path were reduced, then the destabilizing tangential force might be reduced. One way of reducing the leakage flow swirl is with the use of ribs or swirl brakes on the housing. In fact, swirl brakes installed upstream of the interstage seal on the Space Shuttle Main Engine High Pressure Oxygen Turbopump completely eliminated the subsynchronous whirl motion over the steady state operating range of the unit (Childs, 1990). The present study investigates the influence of swirl brakes on the rotordynamic forces generated by discharge-to- suction leakage flows in shrouded centrifugal pumps over a range of subsynchronous whirl ratios and various leakage flow rates typical of present rocket turbopump designs. In addition, the effectiveness of swirl brakes in the presence of leakage inlet (pump discharge) swirl is also demonstrated.

\section{ROTORDYNAMIC FORCES}

Figure (1) shows a schematic of the hydrodynamic forces that act on a rotating impeller whirling in a circular orbit. $F^{*}{ }_{x}$ and $F^{*}{ }_{y}$ are the instantaneous lateral forces in the laboratory frame. $\Omega$ is the whirl radian frequency and $\omega$ is the main shaft radian frequency. The eccentricity of the orbit is given by $\varepsilon$. The lateral forces are given in linear form as:

$$
\left(\begin{array}{l}
F_{x}^{*}(t) \\
F_{y}^{*}(t)
\end{array}\right)=\left(\begin{array}{c}
F_{o x}^{*} \\
F_{o y}^{*}
\end{array}\right)+\left[A^{*}\right]\left(\begin{array}{c}
x^{*}(t) \\
y^{*}(t)
\end{array}\right)
$$

$F_{o x}^{*}$ and $F_{o y}^{*}$ are the steady forces which result from flow asymmetries in the volute. $\left[A^{*}\right]$ is the rotordynamic force matrix. It is a function of the mean flow conditions, pump geometry, whirl frequency ratio $\Omega / \omega$ and if outside the linear range it may also be a function of the eccentricity $\varepsilon$. In the case of a circular whirl orbit:

$$
\begin{aligned}
x^{*}(t) & =\varepsilon \cos (\Omega t) \\
y^{*}(t) & =\varepsilon \sin (\Omega t)
\end{aligned}
$$

The normal and tangential forces for a circular whirl orbit are given by (Jery, 1986 and Franz 1989):

$$
\begin{gathered}
F_{n}^{*}(t)=\frac{1}{2}\left(A_{x x}^{*}+A_{y y}^{*}\right) \varepsilon \\
F_{t}^{*}(t)=\frac{1}{2}\left(-A_{x y}^{*}+A_{y x}^{*}\right) \varepsilon
\end{gathered}
$$

\section{ROTORDYNAMIC COEFFICIENTS AND STABILITY}

To study the stability of an impeller, it is convenient for rotordynamicists to fit the dimensionless normal force $F_{n}$ to a quadratic function of the whirl ratio and to fit the dimensionless tangential force $F_{t}$ to a linear function of the whirl ratio. The expressions are given by:

$$
\begin{gathered}
F_{n}=M\left(\frac{\Omega}{\omega}\right)^{2}-c\left(\frac{\Omega}{\omega}\right)-K \\
F_{t}=-C\left(\frac{\Omega}{\omega}\right)+k
\end{gathered}
$$




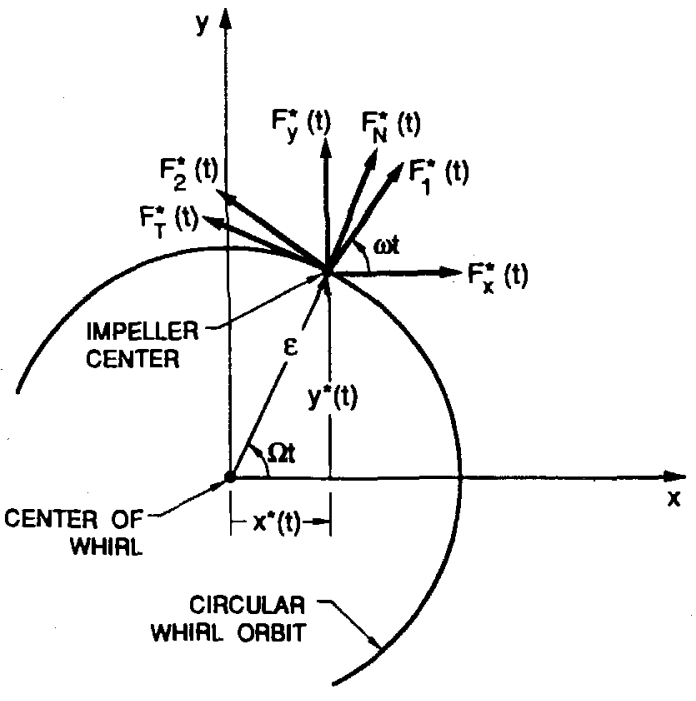

FIGURE 1. SCHEMATIC OF THE FLUID-INDUCED FORCES ACTING ON AN IMPELLER WHIRLING IN A CIRCULAR ORBIT.

where the dimensionless coefficients are the direct added mass (M), direct damping (C), cross-coupled damping (c), direct stiffness ( $\mathrm{K})$, and cross-coupled stiffness (k). As can be seen from equation (7), a positive cross-coupled stiffness is destabilizing because it starts the forward whirl of the impeller since it is equal to the tangential force at zero whirl ratio. Also, from equation (6), a large negative direct stiffness is destabilizing because it promotes a positive normal force which increases the eccentricity of the whirl orbit.

A convenient measure of the rotordynamic stability is the ratio of the cross-coupled stiffness to the direct damping (i.e. $k / C$ ) termed the whirl ratio. This is just a measure of the range of positive whirl frequency ratios for which the tangential force is destabilizing.

\section{TEST APPARATUS}

The present experiments were conducted in the Rotor Force Test Facility (RFTF) at Caltech. The leakage flow test section of the facility is shown in Figure (2).

The working fluid is water. The main components of the test section apparatus consist of a solid or dummy impeller (or rotating shroud), a housing (or stationary shroud) instrumented for pressure measurements, a rotating dynamometer (or internal force balance), an eccentric whirl mechanism (not shown), a leakage exit seal ring and a leakage inlet swirl vane (shown installed in Figure (3)). The solid impeller is used so that leakage

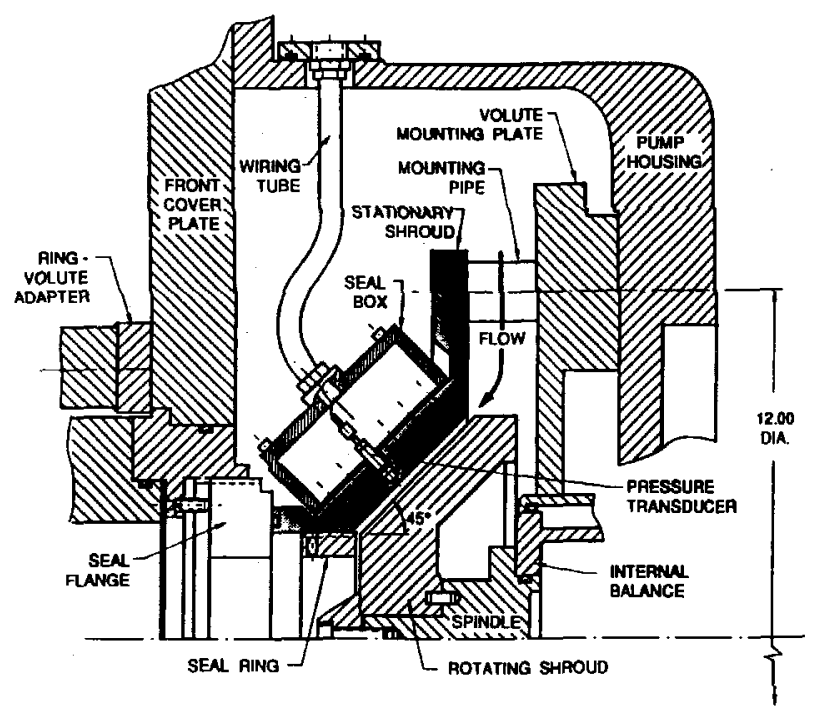

FIGURE 2. LEAKAGE FLOW TEST SECTION (ZHUANG, 1989).

flow contributions to the forces are measured but the main through flow contributions are not experienced. The inner surface of the housing has been modified to accommodate meridional ribs or swirl brakes along the entire length of the leakage annulus. The ribs are each $3 / 16$ of an inch wide and $2 \mathrm{~mm}$ high. Up to 8 equally spaced ribs can be installed. The leakage flow annulus between the impeller and housing is inclined at $45^{\circ}$ to the axis of ratation. The nominal clearance between the solid impeller and the housing can be varied by axial adjustment of the housing. The flow through the leakage path is generated by an auxiliary pump. The solid impeller is mounted on a spindel attached to the rotating dynamometer connected to a data acquisition system which permits measurements of the rotordynamic force matrix. Jery, 1986 and Franz, 1989 describe the operation of the dynamometer. The eccentric drive mechanism imposes a circular whirl orbit on the basic main shaft rotation. The radius of the circular whirl orbit (or eccentricity) can be varied. The seal ring at the leakage exit models a wear ring. The clearance between the seal ring and impeller face is adjustable. The effect of swirl was investigated by installing a swirl vane at the leakage inlet to introduce pre-rotated fluid in the direction of shaft rotation. Figure (3) shows that the vane consists of a logarithmic spiral channel with a turning angle of $2^{\circ}$. The swirl ratio $\Gamma$ (the ratio of the leakage flow circumferential velocity to the impeller tip velocity) is varied by changing the leakage flow rate. The 


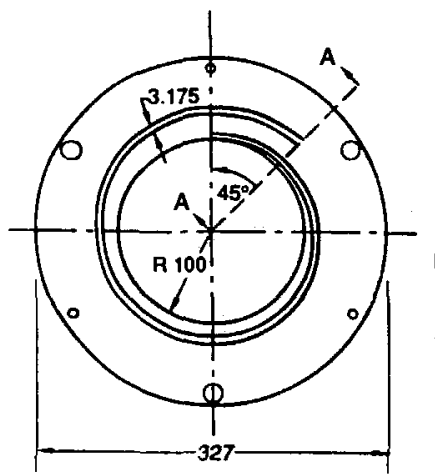

FRONT VIEW

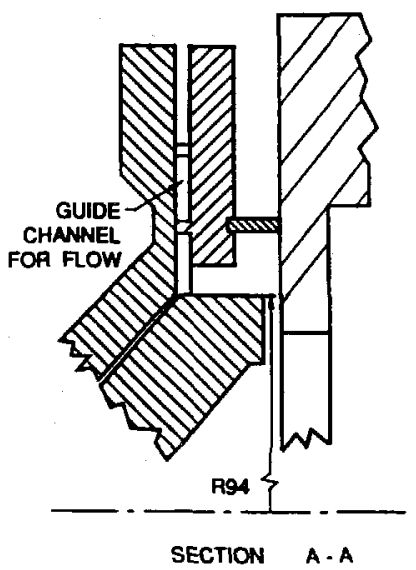

FIGURE 3. INSTALLATION OF LEAKAGE INLET SWIRL VANE. DIMENSIONS IN MM (GUINZBURG, 1992b).

swirl ratio depends on the flow coefficient according to:

$$
\frac{\Gamma}{\phi}=\frac{H}{B \operatorname{Tan}(\alpha)}
$$

$B$ is the depth of the logarithmic spiral channel equal to 0.125 in. A derivation of equation (8) can be found in Guinzburg, 1992a.

\section{TEST MATRIX}

This experiment is designed to measure the rotordynamic forces due to simulated leakage flows for different parameters such as whirl frequency ratio, shaft speed, leakage flow coefficient, number of swirl brake ribs and leakage inlet swirl ratio. For all tests a nominal annulus clearance $H$ of 0.167 in, a whirl eccentricity $\varepsilon$ of 0.0465 in and a leakage exit face seal clearance of 0.04 in were maintained. Tests without leakage inlet swirl were conducted at shaft speeds $\omega$ of 1000 and 2000 RPM, leakage flow rates $Q$ of $0,10,20$ and 30 GPM and 0,4 and 8 brake ribs. For the 1000 RPM runs, the above flow rates correspond to flow coefficients $\phi$ of $0.0,0.026,0.052$ and 0.077 , respectively. For the 2000 RPM runs, the above flow rates correspond to flow coefficients $\phi$ of $0.0,0.013,0.026$ and 0.039 , repectively. The above flow coefficients cover the range of typical leakage rates for the new Space Shuttle Alternate Turbopump (ATP) presently being developed. For the 1000 RPM runs, tests are performed for whirl frequency ratios in the range $-0.9 \leq \frac{\Omega}{\omega} \leq+0.9$ at 0.1 increments.
For the 2000 RPM runs, tests are performed for whirl frequency ratios in the range $-0.6 \leq \frac{\Omega}{\omega} \leq+0.7$ at 0.1 increments. Tests with the swirl vane installed are conducted at a shaft speed of 2000 RPM and the same flow rates as above. With the swirl vane, the above leakage flow rates yield swirl ratios $\Gamma$ of $0.0,0.5,1.0$ and 1.5 , respectively. The above test matrix is summarized in Tables 1 and 2.

TABLE 1. TESTS WITHOUT SWIRL

\begin{tabular}{|c|c|c|c|c|}
\hline RPM & $\Omega / \omega$ & Brakes & $Q(G P M)$ & $\phi$ \\
\hline \multirow[t]{3}{*}{1000} & -0.9 to +0.9 & 0 & $\begin{array}{l}0 \\
10 \\
20 \\
30\end{array}$ & $\begin{array}{l}0.0 \\
0.026 \\
0.052 \\
0.077\end{array}$ \\
\hline & & 4 & $\begin{array}{l}0 \\
10 \\
20 \\
30\end{array}$ & $\begin{array}{l}0.0 \\
0.026 \\
0.052 \\
0.077\end{array}$ \\
\hline & & 8 & $\begin{array}{l}0 \\
10 \\
20 \\
30\end{array}$ & $\begin{array}{l}0.0 \\
0.026 \\
0.052 \\
0.077\end{array}$ \\
\hline \multirow[t]{3}{*}{2000} & -0.6 to +0.7 & 0 & $\begin{array}{l}0 \\
10 \\
20 \\
30\end{array}$ & $\begin{array}{l}0.0 \\
0.013 \\
0.026 \\
0.039\end{array}$ \\
\hline & & 4 & $\begin{array}{l}0 \\
10 \\
20 \\
30\end{array}$ & $\begin{array}{l}0.0 \\
0.013 \\
0.026 \\
0.039\end{array}$ \\
\hline & & 8 & $\begin{array}{l}0 \\
10 \\
20 \\
30\end{array}$ & $\begin{array}{l}0.0 \\
0.013 \\
0.026 \\
0.039\end{array}$ \\
\hline
\end{tabular}

TABLE 2. TESTS WITH SWIRL

\begin{tabular}{|c|c|c|c|c|c|}
\hline RPM & $\Omega / \omega$ & Brakes & $\mathrm{Q}(\mathrm{GPM})$ & $\phi$ & $\Gamma$ \\
\hline \multirow[t]{3}{*}{2000} & -0.6 to +0.7 & 0 & $\begin{array}{l}0 \\
10 \\
20 \\
30\end{array}$ & $\begin{array}{l}0.0 \\
0.013 \\
0.026 \\
0.039\end{array}$ & $\begin{array}{l}0.0 \\
0.5 \\
1.0 \\
1.5\end{array}$ \\
\hline & & 4 & $\begin{array}{l}0 \\
10 \\
20 \\
30\end{array}$ & $\begin{array}{l}0.0 \\
0.013 \\
0.026 \\
0.039\end{array}$ & $\begin{array}{l}0.0 \\
0.5 \\
1.0 \\
1.5\end{array}$ \\
\hline & & 8 & $\begin{array}{l}0 \\
10 \\
20\end{array}$ & $\begin{array}{l}0.0 \\
0.013 \\
0.026\end{array}$ & $\begin{array}{l}0.0 \\
0.5 \\
1.0\end{array}$ \\
\hline
\end{tabular}




\section{RESULTS FOR TESTS WITHOUT LEAKAGE INLET SWIRL}

Figures (4) and (5) show plots of the dimensionless rotordynamic coefficients for 2000 and $1000 \mathrm{RPM}$, respectively for the three different number of brake ribs. Figures (6) and (7) show the whirl ratio. Tests are done at two different rotor speeds to access the Reynolds Number effects. The 2000 RPM runs are shown first since they give the results at a lower flow coefficient range. A comparison of Figures (4) and (5) show differences between corresponding coefficient plots at the lower flow coefficients indicating that Reynolds Number effects are important at low flow rates. At flow coefficients above about 0.03 there seems to be little difference between corresponding coefficient plots at 1000 and 2000 RPM. Reynolds Number effects are stronger with no brake ribs installed. $C$ and $k$ seem to have the greatest discrepencies between the 1000 and 2000 RPM runs.

Focusing on the coefficients corresponding to the tangential force, we can see that with no brakes the tangential force decreases with increasing leakage flow rate, as was found previously by Guinzburg, (1992a). The cross-coupled stiffness coefficient, $k$, is decreased by increasing the flow from 0 to $30 \mathrm{GPM}$, and for the 1000 RPM case, $\mathrm{C}$ remains essentially constant. Hence the range for which the tangential force is destabilizing, $\mathrm{k} / \mathrm{C}$, decreases with increasing flow rate. This can be seen in Figure (7). However, looking at $k$ for the case with 4 or 8 brake ribs, the opposite trend with flow rate is observed. The cross-coupled stiffness increases as the flow rate increases from 0 to 30 GPM in both the 1000 and 2000 RPM runs. The above may appear to indicate that brakes are not beneficial. On the contrary, although the destabilizing tangential force with brakes increases with flow rate, there is a flow rate below which the tangential force with brakes is less than the tangential force without brakes. From the $\mathrm{k}$ plots in Figures (4) and (5) we can see that below $\phi=0.025$ the cross-coupled stiffness coefficient is reduced by the addition of 4 or 8 brakes. 8 brakes are only marginally more effective than 4 . The beneficial effect of brakes is better exemplified by looking at the whirl ratio plots in Figures (6) and (7). Below about $\phi=0.03$, the range of destabilizing tangential force is much less with the addition of brakes.

Focusing on the coefficients corresponding to the normal force, we can see that with no brakes, the normal force increases with increasing leakage flow rate. $\mathrm{K}$ becomes more negative with increasing flow, hence increasing the normal force at zero whirl frequency and therfore is destabilizing. The same is true with 4 or 8 brakes, but we can also see from $\mathrm{K}$ that the destabilizing normal force is significantly reduced by the addition of brakes throughout the range of flow rates tested.
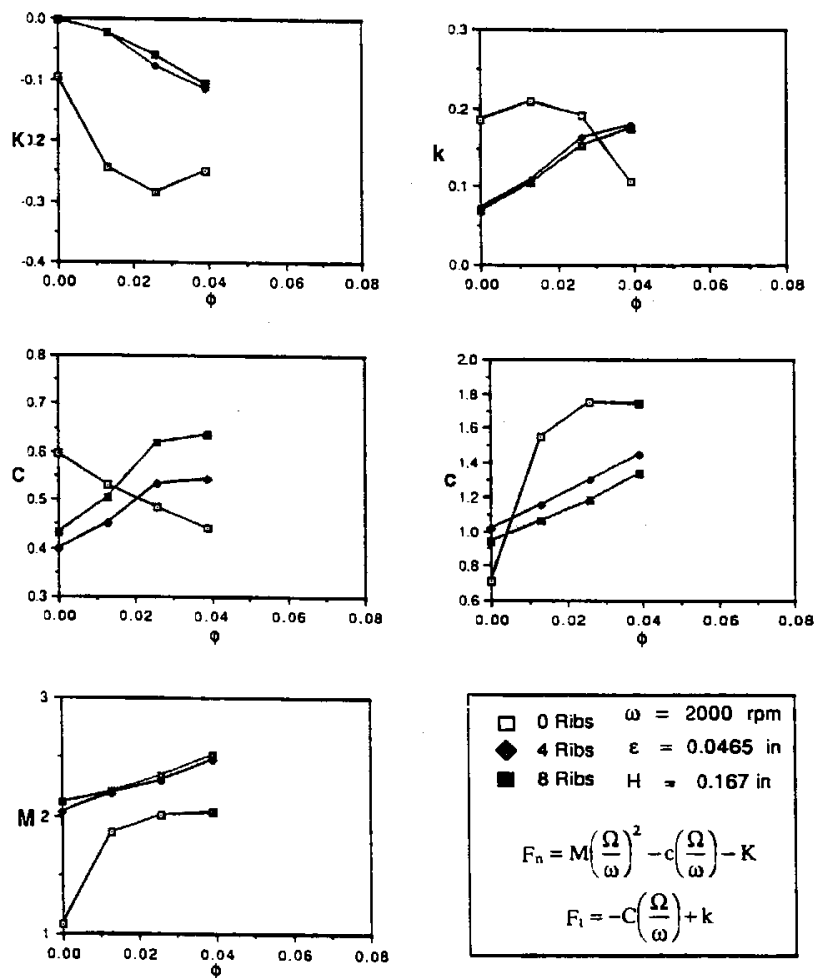

FIGURE 4. ROTORDYNAMIC COEFFICIENTS AT 2000 RPM, NO SWIRL.
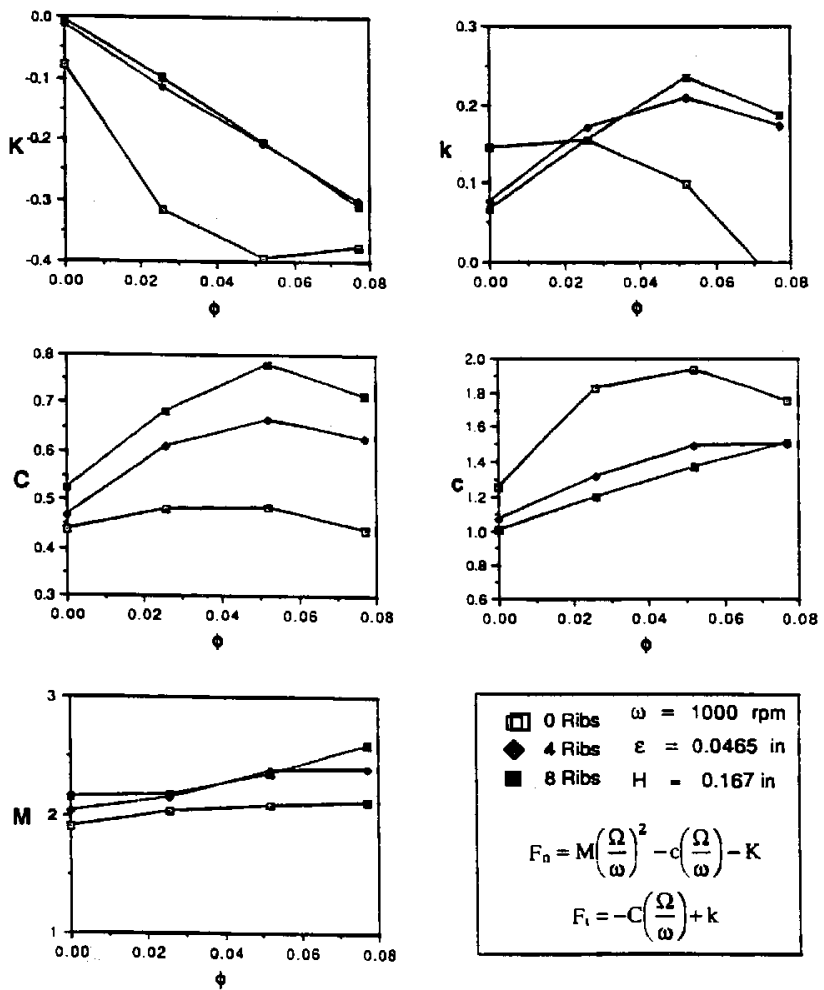

FIGURE 5. ROTORDYNAMIC COEFFICIENTS AT 1000 RPM, NO SWIRL. 


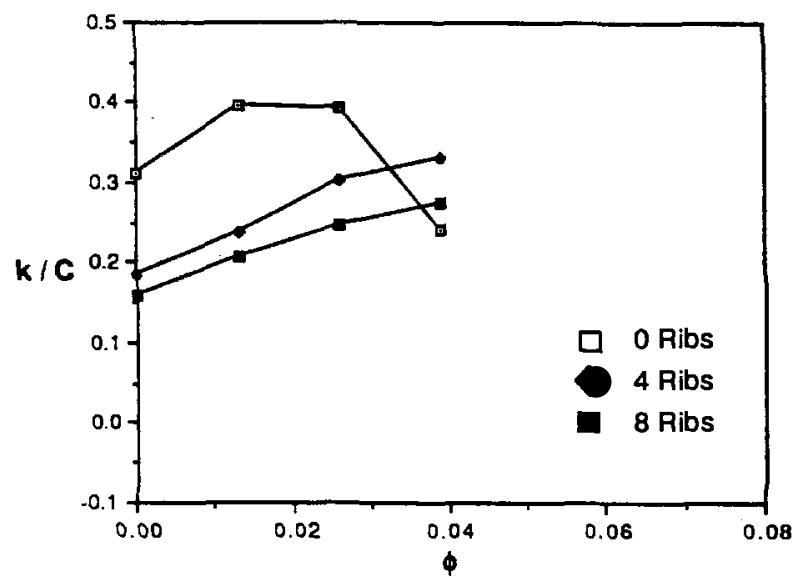

FIGURE 6. WHIRL RATIO AT 2000 RPM, NO SWIRL.

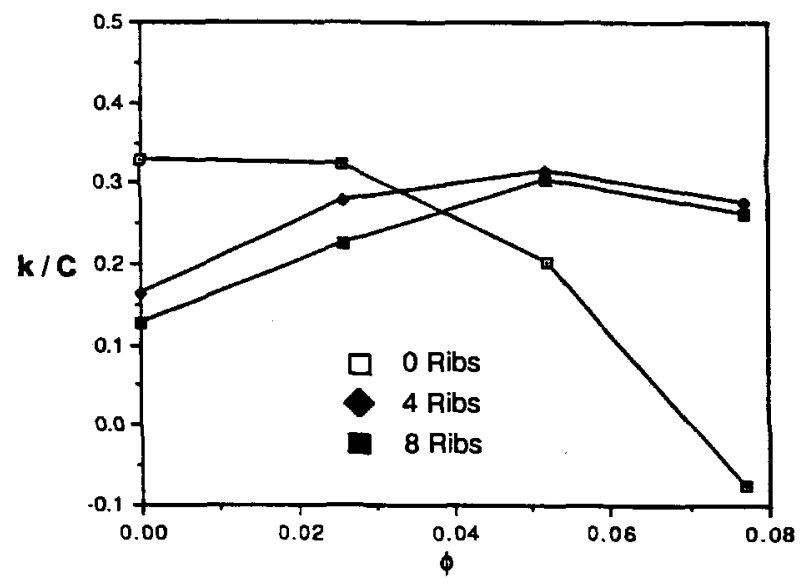

FIGURE 7. WHIRL RATIO AT 1000 RPM, NO SWIRL.

\section{RESULTS FOR TESTS WITH LEAKAGE INLET SWIRL}

Figure (8) shows plots of the dimensionless rotordynamic coefficients for the 2000 RPM runs with leakage inlet swirl for the three different number of ribs. Figure (9) shows the whirl ratio. As was previously found by Guinzburg, (1992b), swirl dramatically increases the tangential force. This is evident by comparing the plot of $k$ without swirl from Figure (4) with the plot of $\mathrm{k}$ with swirl from Figure (8). Once again, with no brakes, it can be seen that as the leakage rate increases, the tangential forces decrease, even though in the process the swirl ratio is increased. The stabilizing effect of an increase in flow rate dominates the destabi- lizing effect of an increase in swirl ratio. When 4 or 8 brake ribs are used, the opposite trend with flow rate is observed, as shown with the results without swirl. With brakes, the tangential force increases with flow rate and swirl ratio. But, for the entire flow range tested, the cross-coupled stiffness is decreased by the addition of brakes.

Focusing on the coefficient $K$ corresponding to the normal force, $\mathbf{K}$ is much large in the presence of swirl. We can see that with no brakes the normal force increases with increasing leakage flow rate. The same is true with 4 or 8 brakes, although from $K$, the destabilizing normal force is significantly reduced by the addition of brakes throughout the range of flow rates tested.

The most dramatic beneficial effect of brakes is found by comparing the whirl ratio plots for 4 or 8 brakes without and with swirl, figures (6) and (9), respectively. The 4 or 8 brake runs with and without swirl are almost identical. This indicates that the brakes almost completely eliminated the effect of swirl. With brakes present, swirl produces only a slight increase in the range for which the tangential force is destabilizing. Also, looking at Figure (9), for all flow rates tested, the addition of brakes reduces the range for which the tangential force is destabilizing.
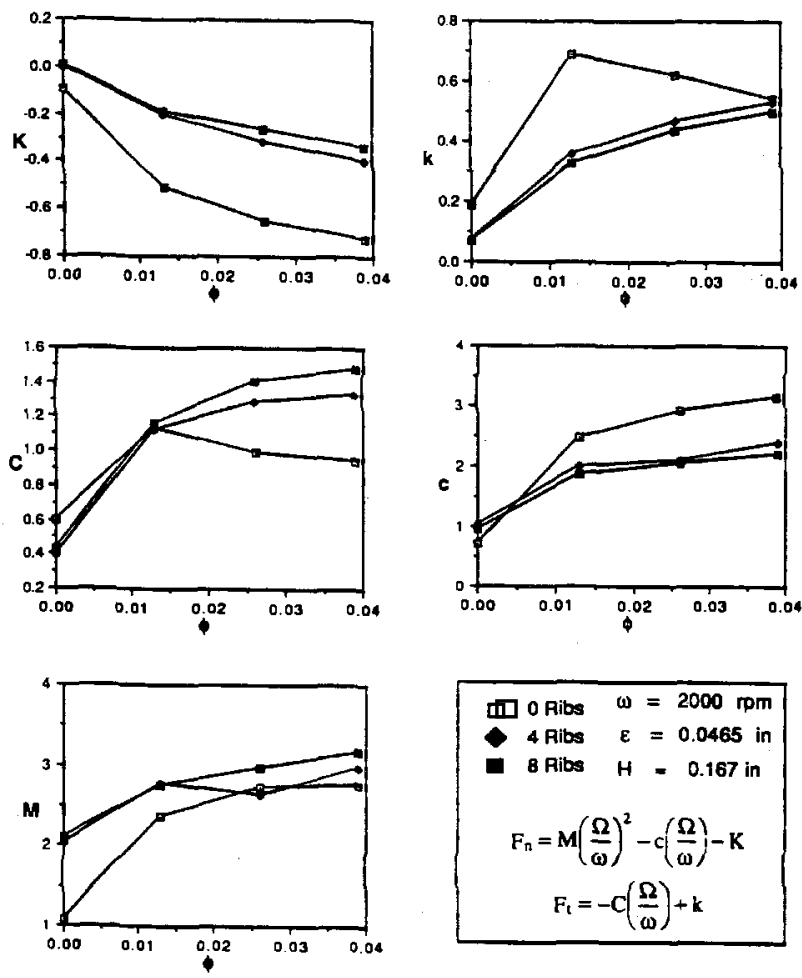

FIGURE 8. ROTORDYNAMIC COEFFICIENTS AT 2000 RPM WITH SWIRL. 


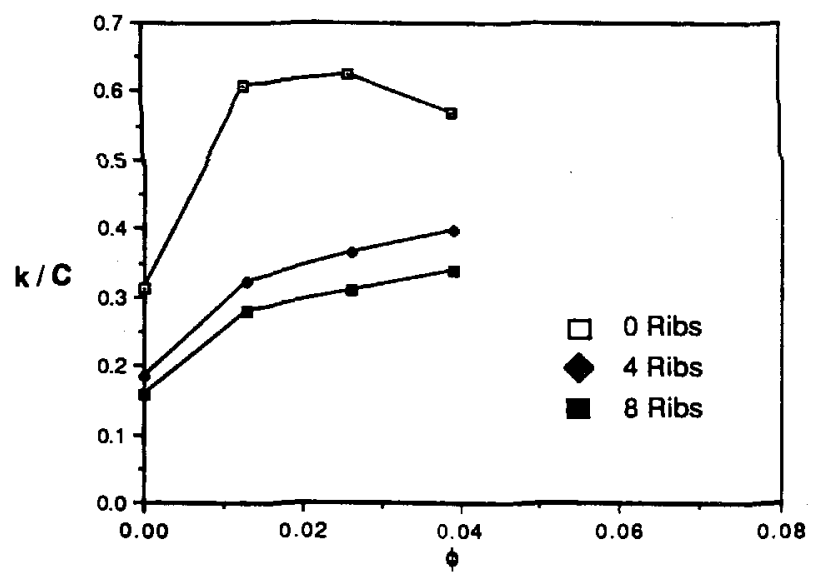

FIGURE 9. WHIRL RATIO AT 2000 RPM WITH SWIRL.

\section{CONCLUSIONS}

The influence of swirl brakes on the rotordynamic forces generated by discharge-to-suction leakage flows in centrifugal pumps with and without leakage inlet swirl has been investigated. The destabilizing normal force at zero whirl is reduced by the addition of brakes. The tangential force and the range of whirl frequency ratios for which the tangential force is destabilizing is reduced by the addition of brakes for low leakage flow rates. Brakes are beneficial in reducing the tangential force up to a higher leakage flow rate in the presence of leakage inlet swirl. Finally, the increase in the range of whirl frequency ratios for which the tangential force is destabilizing due to an increase in the leakage inlet swirl is nearly eliminated by the addition of swirl brakes.

\section{ACKNOWLEDGEMENTS}

The assistance provided by Asif Khalak and Kyle Roof with the experimental program is greatly appreciated. We would also like to thank the NASA George Marshall Space Flight Center for support under Grant NAG8-118 and technical monitor Henry Stinson.

\section{REFERENCES}

Adkins, D. R. and Brennen, C. E., 1988, Analysis of Hydrodynamic Radial Forces on Centrifugal Pump Impellers, ASME J. Fluids Eng., Vol. 110, No. 1, pp. 20-28.
Childs, D. W., Baskharone, E. and Ramsey, C., 1990, Test Results for Rotordynamic Coefficients of the SSME HPOTP Turbine Interstage Seal with Two Swirl Brakes, Trans of ASME, Journal of Tribology, 90-Trib45.

Franz, R. J., 1989, Experimental Investigation of the Effect of Cavitation on the Rotordynamic Forces on a Whirling Centrifugal Pump Impeller, Ph.D. Thesis, California Institute of Technology.

Guinzburg, A., 1992a, Rotordynamic Forces Generated By Discharge-To-Suction Leakage Flows in Centrifugal Pumps, Ph.D. Thesis, California Institute of Technology.

Guinzburg, A., 1992b, The Effect of Inlet Swirl on the Rotordynamic Shroud Forces in a Centrifugal Pump, ASME Paper 92-GT-126.

Jery, B., 1986, Experimental Study of Unsteady Hydrodynamic Force Matrices on Whirling Centrifugal Pump Impellers, Ph.D. Thesis, California Institute of Technology.

Zhuang, F., 1989, Experimental Investigation of the Hydrodynamic Forces on the Shroud of a Centrifugal Pump Impeller, E249.9, Division of Engineering and Applied Science, California Institute of Technology. 